\title{
Mean values of Dirichlet polynomials and applications to linear equations with prime variables
}

\author{
by \\ Stephen Kwok-Kwong Choi (Burnaby) and \\ Angel V. Kumchev (Towson, MD)
}

1. Introduction. In this paper we study Dirichlet polynomials of the form

$$
D(s, \chi)=\sum_{n \leq N} a_{n} \chi(n) n^{-s}
$$

where $\chi(n)$ is a Dirichlet character, $s=\sigma+i t$ is a complex variable, and $a_{n}$ are (complex) coefficients. Such Dirichlet polynomials are an important tool in multiplicative number theory and there is a vast literature on the subject. In particular, one often needs estimates for mean values of the form

$$
\sum_{\chi \in \mathcal{H}} \int_{-T}^{T}\left|\sum_{N<n \leq 2 N} \Lambda(n) \chi(n) n^{-i t}\right| d t
$$

where $\Lambda(n)$ is the von Mangoldt function and the outer summation is over some family of characters, possibly to various moduli. Our main result is Theorem 1.1 below, which deals with the most common types of such averages.

Let $m \geq 1, r \geq 1$, and $Q \geq r$. We consider a set $\mathcal{H}(m, r, Q)$ of characters $\chi=\xi \psi$ modulo $m q$, where $\xi$ is a character modulo $m$ and $\psi$ is a primitive character modulo $q$, with $r \leq q \leq Q, r \mid q$, and $(q, m)=1$. Our result is as follows.

Theorem 1.1. Let $m \geq 1, r \geq 1, Q \geq r, T \geq 2, N \geq 2$, and $\mathcal{H}(m, r, Q)$ be a set of characters as described above. Then

$$
\sum_{\chi \in \mathcal{H}(m, r, Q)} \int_{-T}^{T}\left|\sum_{N<n \leq 2 N} \Lambda(n) \chi(n) n^{-i t}\right| d t \ll\left(N+H N^{11 / 20}\right) L^{C},
$$

2000 Mathematics Subject Classification: Primary 11P05, 11P32, 11P55; Secondary 11L20.

Research of Stephen Choi was supported by NSERC of Canada. 
where $C$ is an absolute constant,

$$
H=m r^{-1} Q^{2} T \quad \text { and } \quad L=\log H N .
$$

REMARK 1. A possible choice for $C$ is $C=1100$, and we have organized the proof as to make this obvious. On the other hand, we have spent no effort to optimize our estimates in that regard, because it is clear that our method will never yield a result with a "respectable" value of $C$, such as $C=10$, or even $C=100$.

Remark 2. Under the Generalized Riemann Hypothesis (GRH), we have

$$
\sum_{\chi \in \mathcal{H}(m, r, Q)} \int_{-T}^{T}\left|\sum_{N<n \leq 2 N} \Lambda(n) \chi(n) n^{-i t}\right| d t \ll N L+H N^{1 / 2} L^{2},
$$

where the term $N L$ on the right side occurs only when the set $\mathcal{H}(m, r, Q)$ contains a principal character. In contrast, because Theorem 1.1 is derived from a general result on bilinear forms (see Theorem 2.1 below), the first term on the right side of (1.2) occurs independent of the presence of a principal character in $\mathcal{H}(m, r, Q)$.

Using Theorem 1.1, we can make progress in an additive problem with prime variables. Consider the linear diophantine equation

$$
a_{1} p_{1}+a_{2} p_{2}+a_{3} p_{3}=b
$$

where $a_{1}, a_{2}, a_{3}, b$ are integers with $a_{1} a_{2} a_{3} \neq 0$ and $p_{1}, p_{2}, p_{3}$ are prime unknowns. Our goal is to prove the existence of solutions of (1.3) which do not grow too rapidly as $B=\max \left\{\left|a_{1}\right|,\left|a_{2}\right|,\left|a_{3}\right|\right\} \rightarrow \infty$. This problem was first raised and investigated by Baker [1] and was later settled, at least qualitatively, by M. C. Liu and Tsang [8]. A necessary condition for the solubility of $(1.3)$ is

$$
a_{1}+a_{2}+a_{3} \equiv b(\bmod 2) .
$$

Without loss of generality, we may also assume that

$$
\left(a_{1}, a_{2}, a_{3}\right)=\left(b, a_{i}, a_{j}\right)=1, \quad 1 \leq i<j \leq 3 .
$$

M. C. Liu and Tsang [8] proved the following result.

THEOREM 1.2. Suppose that $a_{1}, a_{2}, a_{3}, b$ are integers with $a_{1} a_{2} a_{3} \neq 0$ and conditions (1.4) and (1.5) hold. Then there exists an absolute constant $A>0$ such that

(i) if $a_{1}, a_{2}, a_{3}$ are all positive, then (1.3) has solutions in primes whenever $b \gg B^{A}$

(ii) if $a_{1}, a_{2}, a_{3}$ are not all of the same sign, then (1.3) has solutions in primes satisfying

$$
\left|a_{j}\right| p_{j} \ll|b|+B^{A} .
$$


It is not difficult to see that one cannot take the exponent $A$ above arbitrarily small, so it remains to estimate the best possible value of $A$. The first numerical upper bound for $A$ was obtained by Choi [2], who showed that $A \leq 4190$. This bound was subsequently reduced to $A \leq 45$ by M. C. Liu and Wang [9] and to $A \leq 38$ by Li [6]. Furthermore, Choi, M. C. Liu, and Tsang [3] showed that under GRH one has $A \leq 5+\varepsilon$ for any fixed $\varepsilon>0$.

Recently, J. Y. Liu and Tsang [7] showed that when condition (1.5) is replaced by the somewhat more restrictive

$$
\left(a_{i}, a_{j}\right)=\left(b, a_{i}\right)=\left(b, a_{j}\right)=1, \quad 1 \leq i<j \leq 3,
$$

then one can take (essentially) $A=17 / 2$. In the last section of this paper, we obtain the following improvement on their result, thus reducing the value of $A$ further to $A=23 / 3$.

TheOREM 1.3. Suppose that $a_{1}, a_{2}, a_{3}, b$ are integers with $a_{1} a_{2} a_{3} \neq 0$ and conditions (1.4) and (1.7) hold.

(i) If $a_{1}, a_{2}, a_{3}$ are all positive, then (1.3) has solutions in primes whenever

$$
b \gg\left(a_{1} a_{2} a_{3}\right)^{20 / 9} B(\log B)^{26} .
$$

(ii) If $a_{1}, a_{2}, a_{3}$ are not all of the same sign, then (1.3) has solutions in primes satisfying

$$
\left|a_{j}\right| p_{j} \ll|b|+\left(a_{1} a_{2} a_{3}\right)^{20 / 9} B(\log B)^{26} .
$$

REMARK 3. The proof of Theorem 1.2 uses the circle method and the Deuring-Heilbronn phenomenon to treat the major arcs, which need to be taken significantly larger than in classical applications. Under condition (1.7) in place of (1.5), one can show that the possible existence of Siegel zeros does not have special influence, and hence the Deuring-Heilbronn phenomenon can be avoided (see [7, Lemma 3.1]). As a result, better results can be obtained without recourse to the heavy numerical computations needed in $[2,6,9]$.

2. Mean values of products of Dirichlet polynomials. We derive Theorem 1.1 from mean-value estimates for products of Dirichlet polynomials of the form

$$
F(s, \chi)=\prod_{i=1}^{3}\left\{\sum_{N_{i}<n \leq N_{i}^{\prime}} b_{i}(n) \chi(n) n^{-s}\right\} .
$$

We assume that $1 \leq N_{i}<N_{i}^{\prime} \leq 2 N_{i}$ and $X=N_{1} N_{2} N_{3} \geq 10$. We also assume that the coefficients $b_{j}(n)$ are subject to

$$
\left|b_{1}(n)\right| \leq \tau_{\kappa}(n), \quad\left|b_{2}(n)\right| \leq \tau_{\nu}(n), \quad\left|b_{3}(n)\right| \leq 1
$$

for some integers $\kappa, \nu \geq 2$. Here, $\tau_{\kappa}(n)$ denotes the $\kappa$-fold divisor function. The main result of this section is the following theorem. 
Theorem 2.1. Suppose that $F(s, \chi)$ is a Dirichlet polynomial as above and $\mathcal{H}(m, r, Q)$ is a set of characters as in Theorem 1.1 and such that either $\mathcal{H}(m, r, Q)$ contains no principal character, or it contains only the trivial character $\chi^{0}(n)=1$ for all $n \geq 1$. Also, suppose that either

(i) $\max \left(N_{1}, N_{2}\right) \ll X^{11 / 20}$ and $b_{3}(n)=1$ for all $n \leq 2 N_{3}$, or

(ii) $\max \left(N_{1}, N_{2}\right) \ll X^{11 / 20}$ and $N_{3} \ll X^{8 / 35}$.

Then

$$
\sum_{\chi \in \mathcal{H}(m, r, Q)} \int_{-T}^{T}|F(i t, \chi)| d t \ll\left(X+H X^{11 / 20}\right) L^{c(\kappa, \nu)},
$$

where $c(\kappa, \nu)=3 \max \left(\kappa^{2}, \nu^{2}\right)+\kappa+\nu+20, H=m r^{-1} Q^{2} T$, and $L=\log 2 H X$.

The main tool in the proof of Theorem 2.1 are bounds for the cardinality of a well-spaced set of points at which a Dirichlet polynomial of the form (1.1) is large. In this context, a "point" is an ordered pair $(t, \chi)$, where $t$ is a real number such that $|t| \leq T$ and $\chi$ is a character from $\mathcal{H}(m, r, Q)$. We say that the points $\left(t_{1}, \chi_{1}\right), \ldots,\left(t_{R}, \chi_{R}\right)$ are well-spaced if $\left|t_{i}-t_{j}\right| \geq 1$ whenever $\chi_{i}=\chi_{j}$ and $i \neq j$.

Lemma 2.2. Suppose that $\left(t_{1}, \chi_{1}\right), \ldots,\left(t_{R}, \chi_{R}\right)$ are well-spaced and that for all $j=1, \ldots, R$,

$$
\left|\sum_{n \leq N} a_{n} \chi_{j}(n) n^{-i t_{j}}\right| \geq V
$$

Then

$$
R \ll\left(N V^{-2}+H \min \left\{V^{-2}, N G^{2} V^{-6}\right\}\right) G L^{18},
$$

where $L=\log 2 H N$ and

$$
G=\sum_{n \leq N}\left|a_{n}\right|^{2}
$$

Proof. When $r=1$, the lemma is a direct consequence of [5, Theorems 9.16 and 9.18]. When $r>1$, we need modifications of those results. The modifications, however, are straightforward because of the following observations:

- the trivial bound for the cardinality of $\mathcal{H}(m, r, Q)$ is

$$
|\mathcal{H}(m, r, Q)| \ll m r^{-1} Q^{2} ;
$$

- if $1 \leq q_{1}, q_{2} \leq Q$ and $r \mid\left(q_{1}, q_{2}\right)$, then $\left[q_{1}, q_{2}\right] \leq r^{-1} Q^{2}$.

Lemma 2.3. Let $N<M \leq c N$ and define

$$
D(s, \chi)=\sum_{N<n \leq M} \chi(n) n^{-s} .
$$


Suppose that $\left(t_{1}, \chi_{1}\right), \ldots,\left(t_{R}, \chi_{R}\right)$ are well-spaced and that $\left|t_{j}\right| \geq N$ whenever $\chi_{j}$ is principal. Then

$$
\sum_{j=1}^{R}\left|D\left(i t_{j}, \chi_{j}\right)\right|^{4} \ll H N^{2} L^{10} .
$$

Proof. Without loss of generality we may assume that the distances from $M$ and $N$ to $\mathbb{Z}$ equal $1 / 2$. For any character $\chi \in \mathcal{H}(m, r, Q)$, Perron's formula (see [5, Proposition 5.54]) yields

$$
D(i t, \chi)=\frac{1}{2 \pi i} \int_{\alpha-i T_{1}}^{\alpha+i T_{1}} L(i t+w, \chi) \frac{M^{w}-N^{w}}{w} d w+O(1),
$$

where $T_{1}=10 H N$ and $\alpha=1+\left(\log T_{1}\right)^{-1}$. The integrand is holomorphic everywhere except possibly at $w=1-i t$, where $L(i t+w, \chi)$ has a simple pole if $\chi$ is principal. Thus, we can move the integration to the contour $\mathfrak{C}$ consisting of the other three sides of the rectangle with vertices $1 / 2 \pm i T_{1}$, $\alpha \pm i T_{1}$. By the convexity bound

$$
L(\sigma+i t, \chi) \ll(m q(|t|+2))^{(1-\sigma) / 2+\varepsilon} \quad(0 \leq \sigma \leq 1),
$$

the integrals over the horizontal parts of $\mathfrak{C}$ contribute at most

$$
\sup _{1 / 2 \leq \sigma \leq \alpha}\left\{T_{1}^{-1} N^{\sigma}\left(m q T_{1}\right)^{(1-\sigma) / 2+\varepsilon}\right\} \ll 1 .
$$

Also, the residue at $w=1-i t$ is $\ll \delta_{\chi} N(1+|t|)^{-1}$, where $\delta_{\chi}$ is 1 or 0 according as $\chi$ is principal or not. Hence, for any point $\left(t_{j}, \chi_{j}\right), j=1, \ldots, R$, we have

$$
\begin{aligned}
D\left(i t_{j}, \chi_{j}\right) & \ll N^{1 / 2} \int_{-T_{1}}^{T_{1}}\left|L\left(1 / 2+i\left(t_{j}+u\right), \chi_{j}\right)\right| \frac{d u}{1+|u|}+\frac{\delta_{\chi_{j}} N}{1+\left|t_{j}\right|}+1 \\
& \ll N^{1 / 2} \int_{-T_{1}}^{T_{1}}\left|L\left(1 / 2+i\left(t_{j}+u\right), \chi_{j}\right)\right| \frac{d u}{1+|u|}+1,
\end{aligned}
$$

where the last inequality uses the hypothesis on points $\left(t_{j}, \chi_{j}\right)$ with principal characters. Appealing to Hölder's inequality, we derive the estimate

$$
\begin{aligned}
\left|D\left(i t_{j}, \chi_{j}\right)\right|^{4} & \ll N^{2} L^{3} \int_{-T_{1}}^{T_{1}}\left|L\left(1 / 2+i\left(t_{j}+u\right), \chi_{j}\right)\right|^{4} \frac{d u}{1+|u|}+1 \\
& \ll N^{2} L^{3} \int_{-2 T_{1}}^{2 T_{1}}\left|L\left(1 / 2+i u, \chi_{j}\right)\right|^{4} \frac{d u}{1+\left|u-t_{j}\right|}+1,
\end{aligned}
$$


whence

$$
\sum_{j=1}^{R}\left|D\left(i t_{j}, \chi_{j}\right)\right|^{4} \ll N^{2} L^{3} \sum_{j=1}^{R} \int_{-2 T_{1}}^{2 T_{1}}\left|L\left(1 / 2+i u, \chi_{j}\right)\right|^{4} \frac{d u}{1+\left|u-t_{j}\right|}+R .
$$

This suffices, because

$$
\begin{aligned}
\sum_{j=1}^{R} \int_{-2 T_{1}}^{2 T_{1}}\left|L\left(1 / 2+i u, \chi_{j}\right)\right|^{4} & \frac{d u}{1+\left|u-t_{j}\right|} \\
& \ll \sum_{\chi \in \mathcal{H}(m, r, Q)} \int_{-2 T_{1}}^{2 T_{1}}|L(1 / 2+i u, \chi)|^{4}\left\{\sum_{\substack{j=1 \\
\chi_{j}=\chi}}^{R} \frac{1}{1+\left|u-t_{j}\right|}\right\} d u \\
& \ll T L \sum_{\chi \in \mathcal{H}(m, r, Q)} \int_{-2 T_{1}}^{2 T_{1}}|L(1 / 2+i u, \chi)|^{4} \frac{d u}{T+|u|} \ll H L^{7},
\end{aligned}
$$

where the final step uses the estimate for the fourth power moment of $L(s, \chi)$ (see [10, Theorem 10.1]).

Proof of Theorem 2.1. Define the Dirichlet polynomials

$$
F_{i}(s, \chi)=\sum_{N_{i}<n \leq N_{i}^{\prime}} b_{i}(n) \chi(n) n^{-s} \quad(i=1,2,3) .
$$

The proof is divided into four steps.

STEP 1. First, we treat the case where $\max \left(N_{1}, N_{2}\right) \geq X^{9 / 20}$. Suppose that $N_{1} \geq N_{2}$. We recall the well known estimate (see [5, (1.80)])

$$
\sum_{n \leq x} \tau_{\kappa}(n)^{\nu} \ll x(\log x)^{\kappa^{\nu}-1} .
$$

By [5, Theorem 9.12] and (2.2),

$$
\sum_{\chi \in \mathcal{H}(m, r, Q)} \int_{-T}^{T}\left|F_{1}(i t, \chi)\right|^{2} d t \ll\left(N_{1}+H\right) N_{1} L^{\kappa^{2}+2} .
$$

Similarly,

$$
\sum_{\chi \in \mathcal{H}(m, r, Q)} \int_{-T}^{T}\left|\widetilde{F}_{2}(i t, \chi)\right|^{2} d t \ll\left(N_{2} N_{3}+H\right) N_{2} N_{3} L^{\nu^{2}+2 \nu+3},
$$

where $\widetilde{F}_{2}(s, \chi)=F_{2}(s, \chi) F_{3}(s, \chi)$ is a Dirichlet polynomial with coefficients $\widetilde{b}_{2}(n)$ subject to

$$
\left|\widetilde{b}_{2}(n)\right| \leq \sum_{n=u v} \tau_{\nu}(u) \leq \tau_{\nu+1}(n)
$$


Using (2.6), (2.7), and the Cauchy-Schwarz inequality, we find that the left side of (2.3) is bounded above by

$$
\begin{aligned}
& \left(X^{1 / 2}+\left(H N_{1}\right)^{1 / 2}+\left(H N_{2} N_{3}\right)^{1 / 2}+H\right) X^{1 / 2} L^{c_{1}} \\
& \ll\left(X+H^{1 / 2} X^{31 / 40}+H X^{1 / 2}\right) L^{c_{1}} \ll\left(X+H X^{11 / 20}\right) L^{c_{1}},
\end{aligned}
$$

where $c_{1}=c_{1}(\kappa, \nu)=\kappa^{2}+\nu^{2}+4$. Since an obvious modification of this argument establishes (2.3) when $N_{2} \geq N_{1}$, we may assume for the remainder of the proof that

$$
\max \left(N_{1}, N_{2}\right) \leq X^{9 / 20} .
$$

SteP 2. Suppose that hypothesis (ii) holds. By a standard argument,

$$
\sum_{\chi \in \mathcal{H}(m, r, Q)} \int_{-T}^{T}|F(i t, \chi)| d t \ll \sum_{j=1}^{R}\left|F\left(i t_{j}, \chi_{j}\right)\right|
$$

where $\left(t_{1}, \chi_{1}\right), \ldots,\left(t_{R}, \chi_{R}\right)$ are well-spaced points. The points $\left(t_{j}, \chi_{j}\right)$ such that

$$
F_{i}\left(i t_{j}, \chi_{j}\right) \ll X^{-1} \quad \text { for some } i=1,2,3
$$

contribute at most

$$
R X^{-1} X^{1.01} \ll R X^{0.01} \ll H X^{0.01}
$$

to the right side of (2.9). We divide the remaining points $\left(t_{j}, \chi_{j}\right)$ into $O\left(L^{3}\right)$ subsets so that for the points in a particular subset $\mathcal{S}\left(V_{1}, V_{2}, V_{3}\right)$ we have

$$
V_{i} \leq\left|F_{i}\left(i t_{j}, \chi_{j}\right)\right| \leq 2 V_{i} \quad(i=1,2,3) .
$$

We obtain

$$
\sum_{\chi \in \mathcal{H}(m, r, Q)} \int_{-T}^{T}|F(i t, \chi)| d t \ll L^{3} V_{1} V_{2} V_{3}\left|\mathcal{S}\left(V_{1}, V_{2}, V_{3}\right)\right|+H X^{0.01}
$$

for some $V_{1}, V_{2}, V_{3}$ subject to

$$
X^{-1} \leq V_{i} \leq N_{i} L^{\kappa+\nu} .
$$

Thus, it suffices to show that

$$
\Xi\left|\mathcal{S}\left(V_{1}, V_{2}, V_{3}\right)\right| \ll\left(X+H X^{11 / 20}\right) L^{c_{2}+\kappa+\nu},
$$

where $\Xi=V_{1} V_{2} V_{3}, c_{2}=c_{2}(\kappa, \nu)=3 \max \left(\kappa^{2}, \nu^{2}\right)+15$. To derive this bound, we apply Lemma 2.2 to $F_{1}(s, \chi), F_{2}(s, \chi)$, and $F_{3}(s, \chi)^{2}$ and find that

$$
\begin{aligned}
\left|\mathcal{S}\left(V_{1}, V_{2}, V_{3}\right)\right| \ll \min \{ & N_{1}^{2} V_{1}^{-2}+H N_{1} \min \left(V_{1}^{-2}, N_{1}^{3} V_{1}^{-6}\right), \\
& N_{2}^{2} V_{2}^{-2}+H N_{2} \min \left(V_{2}^{-2}, N_{2}^{3} V_{2}^{-6}\right), \\
& \left.N_{3}^{4} V_{3}^{-4}+H N_{3}^{2} \min \left(V_{3}^{-4}, N_{3}^{6} V_{3}^{-12}\right)\right\} L^{c_{2} .}
\end{aligned}
$$


We now derive (2.13) from (2.8), (2.12), and (2.14) using a case-by-case analysis. We write

$$
\Gamma_{i}=N_{i}^{2} V_{i}^{-2}, \quad \Delta_{i}=\min \left(V_{i}^{-2}, N_{i}^{3} V_{i}^{-6}\right), \quad \Delta_{i}(\alpha)=N_{i}^{3 \alpha} V_{i}^{-2-4 \alpha}
$$

and remark that $\Delta_{i} \leq \Delta_{i}(\alpha)$ for all $0 \leq \alpha \leq 1$.

CASE 1: $\Gamma_{1} \geq H N_{1} \Delta_{1}$ and $\Gamma_{2} \geq H N_{2} \Delta_{2}$. Then, by (2.12) and (2.14),

$$
\begin{aligned}
\Xi\left|\mathcal{S}\left(V_{1}, V_{2}, V_{3}\right)\right| & \ll \Xi \min \left\{\Gamma_{1}, \Gamma_{2}\right\} L^{c_{2}} \ll \Xi\left(\Gamma_{1} \Gamma_{2}\right)^{1 / 2} L^{c_{2}} \\
& \ll N_{1} N_{2} V_{3} L^{c_{2}} \ll X L^{c_{2}+\kappa+\nu} .
\end{aligned}
$$

CASE 2: $\Gamma_{1} \leq H N_{1} \Delta_{1}, \Gamma_{2} \leq H N_{2} \Delta_{2}$, and $\Gamma_{3}^{2} \geq H N_{3}^{2} \Delta_{3}^{2}$. By $(2.14)$ and the hypothesis $N_{3} \leq X^{8 / 35}$, we get

$$
\begin{aligned}
\Xi\left|\mathcal{S}\left(V_{1}, V_{2}, V_{3}\right)\right| & \ll \Xi \min \left\{H N_{1} \Delta_{1}, H N_{2} \Delta_{2}, \Gamma_{3}^{2}\right\} L^{c_{2}} \\
& \ll \Xi\left(H N_{1} \Delta_{1}(1 / 6)\right)^{3 / 8}\left(H N_{2} \Delta_{2}(1 / 6)\right)^{3 / 8} \Gamma_{3}^{1 / 2} L^{c_{2}} \\
& \ll H^{3 / 4}\left(X^{9} N_{3}^{7}\right)^{1 / 16} L^{c_{2}} \ll\left(X+H X^{11 / 20}\right) L^{c_{2}},
\end{aligned}
$$

where the last step uses the fact that

$$
H^{3 / 4}\left(X^{9} N_{3}^{7}\right)^{1 / 16} \ll H^{3 / 4} X^{53 / 80}=X^{1 / 4}\left(H X^{11 / 20}\right)^{3 / 4} .
$$

CASE 3: $\Gamma_{1} \leq H N_{1} \Delta_{1}, \Gamma_{2} \leq H N_{2} \Delta_{2}$, and $\Gamma_{3}^{2} \leq H N_{3}^{2} \Delta_{3}^{2}$. When $N_{3} \leq$ $X^{1 / 5}$, then (2.14) yields

$$
\begin{aligned}
\Xi\left|\mathcal{S}\left(V_{1}, V_{2}, V_{3}\right)\right| & \ll \Xi \min \left\{H N_{1} \Delta_{1}, H N_{2} \Delta_{2}, H N_{3}^{2} \Delta_{3}^{2}\right\} L^{c_{2}} \\
& \ll \Xi H\left(N_{1} \Delta_{1}(1 / 22) N_{2} \Delta_{2}(1 / 22)\right)^{11 / 24}\left(N_{3} \Delta_{3}(1)\right)^{1 / 6} L^{c_{2}} \\
& \ll H\left(X^{25} N_{3}^{7}\right)^{1 / 48} L^{c_{2}} \ll H X^{11 / 20} L^{c_{2}} .
\end{aligned}
$$

On the other hand, when $N_{3} \geq X^{1 / 5},(2.14)$ yields

$$
\begin{aligned}
\Xi\left|\mathcal{S}\left(V_{1}, V_{2}, V_{3}\right)\right| & \ll \Xi H\left(N_{1} \Delta_{1}(1 / 6) N_{2} \Delta_{2}(1 / 6)\right)^{3 / 8}\left(N_{3} \Delta_{3}(0)\right)^{1 / 2} L^{c_{2}} \\
& \ll H\left(X^{9} N_{3}^{-1}\right)^{1 / 16} L^{c_{2}} \ll H X^{11 / 20} L^{c_{2}} .
\end{aligned}
$$

CASE 4: $\Gamma_{1} \geq H N_{1} \Delta_{1}, \Gamma_{2} \leq H N_{2} \Delta_{2}$, and $\Gamma_{3}^{2} \geq H N_{3}^{2} \Delta_{3}^{2}$. By (2.8), (2.14), and the hypothesis $N_{3} \leq X^{8 / 35}$,

$$
\begin{aligned}
\Xi\left|\mathcal{S}\left(V_{1}, V_{2}, V_{3}\right)\right| & \ll \Xi \min \left\{\Gamma_{1}, H N_{2} \Delta_{2}, \Gamma_{3}^{2}\right\} L^{c_{2}} \\
& \ll \Xi\left(\Gamma_{1} \Gamma_{3}\right)^{1 / 2}\left(H N_{2} \Delta_{2}(1 / 2)\right)^{1 / 4} L^{c_{2}} \\
& \ll H^{1 / 4} X^{5 / 8}\left(N_{1} N_{3}\right)^{3 / 8} L^{c_{2}} \ll\left(X+H X^{11 / 20}\right) L^{c_{2}},
\end{aligned}
$$

where the last step uses the fact that

$$
H^{1 / 4} X^{5 / 8}\left(N_{1} N_{3}\right)^{3 / 8} \ll H^{1 / 4} X^{197 / 224} \ll X^{3 / 4}\left(H X^{11 / 20}\right)^{1 / 4} .
$$


CASE 5: $\Gamma_{1} \geq H N_{1} \Delta_{1}, \Gamma_{2} \leq H N_{2} \Delta_{2}$, and $\Gamma_{3}^{2} \leq H N_{3}^{2} \Delta_{3}^{2}$. When $N_{3} \leq$ $X^{1 / 5}$, then (2.14) yields

$$
\begin{aligned}
\Xi\left|\mathcal{S}\left(V_{1}, V_{2}, V_{3}\right)\right| & \ll \Xi \min \left\{\Gamma_{1}, H N_{2} \Delta_{2}, H N_{3}^{2} \Delta_{3}^{2}\right\} L^{c_{2}} \\
& \ll \Xi \Gamma_{1}^{1 / 2}\left(H N_{2} \Delta_{2}(1 / 10)\right)^{5 / 12}\left(H N_{3}^{2} \Delta_{3}(1)^{2}\right)^{1 / 12} L^{c_{2}} \\
& \ll H^{1 / 2}\left(N_{1}^{11} N_{3}^{3}\right)^{1 / 24} X^{13 / 24} L^{c_{2}} \ll\left(X+H X^{11 / 20}\right) L^{c_{2}},
\end{aligned}
$$

where the last step uses the fact that

$$
H^{1 / 2}\left(N_{1}^{11} N_{3}^{3}\right)^{1 / 24} X^{13 / 24} \ll H^{1 / 2} X^{371 / 480} \ll X^{1 / 2}\left(H X^{11 / 20}\right)^{1 / 2} .
$$

On the other hand, when $N_{3} \geq X^{1 / 5}$, by (2.14),

$$
\begin{aligned}
\Xi\left|\mathcal{S}\left(V_{1}, V_{2}, V_{3}\right)\right| & \ll \Xi \Gamma_{1}^{1 / 2}\left(H N_{2} \Delta_{2}(1 / 2)\right)^{1 / 4}\left(H N_{3}^{2} \Delta_{3}(0)^{2}\right)^{1 / 4} L^{c_{2}} \\
& \ll H^{1 / 2}\left(X^{5} N_{1}^{3} N_{3}^{-1}\right)^{1 / 8} L^{c_{2}} \ll\left(X+H X^{11 / 20}\right) L^{c_{2}},
\end{aligned}
$$

because

$$
H^{1 / 2}\left(X^{5} N_{1}^{3} N_{3}^{-1}\right)^{1 / 8} \ll H^{1 / 2} X^{123 / 160} \ll X^{1 / 2}\left(H X^{11 / 20}\right)^{1 / 2} .
$$

CAse 6: $\Gamma_{1} \leq H N_{1} \Delta_{1}$ and $\Gamma_{2} \geq H N_{2} \Delta_{2}$. This case can be split into two subcases that can be handled similarly to Cases 4 and 5 .

This completes the proof under hypothesis (ii).

STEP 3. Suppose that hypothesis (i) holds and $\mathcal{H}(m, r, Q)$ contains no principal characters. If $N_{3} \leq X^{8 / 35}$, hypothesis (i) implies hypothesis (ii), so we may assume that $N_{3} \geq X^{8 / 35}$. Then we follow the argument from Step 2 until we reach (2.12). Under the present assumptions, Lemma 2.3 and (2.10) yield

$$
\left|\mathcal{S}\left(V_{1}, V_{2}, V_{3}\right)\right| \ll H N_{3}^{2} V_{3}^{-4} L^{10} .
$$

Thus, we may replace (2.14) by the bound

$$
\begin{aligned}
\left|\mathcal{S}\left(V_{1}, V_{2}, V_{3}\right)\right| \ll & \min \left\{N_{1}^{2} V_{1}^{-2}+H N_{1} \min \left(V_{1}^{-2}, N_{1}^{3} V_{1}^{-6}\right),\right. \\
& \left.N_{2}^{2} V_{2}^{-2}+H N_{2} \min \left(V_{2}^{-2}, N_{2}^{3} V_{2}^{-6}\right), H N_{3}^{2} V_{3}^{-4}\right\} L^{c_{2}} .
\end{aligned}
$$

We now embark on deriving (2.13) from (2.8), (2.12), (2.15), and the hypothesis $N_{3} \geq X^{8 / 35}$. Let $\Gamma_{i}$ and $\Delta_{i}$ be as in Step 2. We consider four cases.

CASE 1: $\Gamma_{1} \geq H N_{1} \Delta_{1}$ and $\Gamma_{2} \geq H N_{2} \Delta_{2}$. Then we argue as in Case 1 of Step 2.

CASE 2: $\Gamma_{1} \leq H N_{1} \Delta_{1}$ and $\Gamma_{2} \leq H N_{2} \Delta_{2}$. Then (2.15) yields

$$
\begin{aligned}
\Xi\left|\mathcal{S}\left(V_{1}, V_{2}, V_{3}\right)\right| & \ll \Xi H\left(N_{1} \Delta_{1}(1 / 6) N_{2} \Delta_{2}(1 / 6)\right)^{3 / 8}\left(N_{3} V_{3}^{-2}\right)^{1 / 2} L^{c_{2}} \\
& \ll H\left(X^{9} N_{3}^{-1}\right)^{1 / 16} L^{c_{2}} \ll H X^{11 / 20} L^{c_{2}} .
\end{aligned}
$$


CASE 3: $\Gamma_{1} \geq H N_{1} \Delta_{1}$ and $\Gamma_{2} \leq H N_{2} \Delta_{2}$. By (2.15),

$$
\begin{aligned}
\Xi\left|\mathcal{S}\left(V_{1}, V_{2}, V_{3}\right)\right| & \ll \Xi \Gamma_{1}^{1 / 2}\left(H N_{2} \Delta_{2}(1 / 2)\right)^{1 / 4}\left(H N_{3}^{2} V_{3}^{-4}\right)^{1 / 4} L^{c_{2}} \\
& \ll H^{1 / 2}\left(X^{5} N_{1}^{3} N_{3}^{-1}\right)^{1 / 8} L^{c_{2}} \ll\left(X+H X^{11 / 20}\right) L^{c_{2}},
\end{aligned}
$$

because

$$
H^{1 / 2}\left(X^{5} N_{1}^{3} N_{3}^{-1}\right)^{1 / 8} \ll H^{1 / 2} X^{857 / 1120} \ll X^{1 / 2}\left(H X^{11 / 20}\right)^{1 / 2} .
$$

CASE 4: $\Gamma_{1} \leq H N_{1} \Delta_{1}$ and $\Gamma_{2} \geq H N_{2} \Delta_{2}$. This is similar to Case 3 .

STEP 4. Finally, suppose that hypothesis (i) holds and $\mathcal{H}(m, r, Q)$ contains only the trivial character $\chi^{0}(n)$. Again, we may assume that $N_{3} \geq$ $X^{8 / 35}$. By [5, Corollary 8.11],

$$
\sum_{N<n \leq 2 N} n^{-i t} \ll N(1+|t|)^{-1} \quad(|t|<N) .
$$

Using this bound and the trivial estimates for the sums over $n_{1}$ and $n_{2}$, we get

$$
\int_{-N_{3}}^{N_{3}}\left|F\left(i t, \chi^{0}\right)\right| d t \ll N_{1} N_{2} L^{\kappa+\nu-2} \int_{-N_{3}}^{N_{3}} \frac{N_{3}}{1+|t|} d t \ll X L^{\kappa+\nu-1} .
$$

On the other hand, when $N_{3} \leq T$, we may repeat Steps 1 and 3 above to deduce that

$$
\int_{N_{3} \leq|t| \leq T}\left|F\left(i t, \chi^{0}\right)\right| d t \ll\left(X+T X^{11 / 20}\right) L^{c_{3}},
$$

where $c_{3}=c_{3}(\kappa, \nu)=3 \max \left(\kappa^{2}, \nu^{2}\right)+\kappa+\nu+15$. The only change occurs when we have to appeal to Lemma 2.3 to obtain a variant of (2.15). Because of the presence of a principal character, we now need to ensure that the points in $\mathcal{S}\left(V_{1}, V_{2}, V_{3}\right)$ satisfy $\left|t_{j}\right| \geq N_{3}$. For the points appearing in the proof of (2.16), however, this condition obviously holds.

We conclude this section with a technical lemma, which will be needed in the next section.

Lemma 2.4. Suppose that $2 \leq T \leq M<N$ and $f: \mathbb{N}^{2} \rightarrow \mathbb{C}$ is a function such that

$$
\sum_{m} \int_{-U}^{U}\left|\sum_{n \leq N} f(m, n) n^{i t}\right| d t \leq A+B U
$$

for all $U \geq 2$. Then

$$
\sum_{m} \int_{-T}^{T}\left|\sum_{n \leq M} f(m, n) n^{i t}\right| d t \ll(A+B T) \log ^{2} N .
$$


Proof. Let $g$ denote the indicator function of $[-T, T]$ and let $h$ be the function constructed in [5, Lemma 13.11] with $z=N$. Then

$$
|h(u)| \ll \min \left\{\log N,|u|^{-1}, N|u|^{-2}\right\}
$$

and

$$
\int_{-\infty}^{\infty} h(u)\left(\frac{m}{n}\right)^{i u} d u= \begin{cases}1 & \text { if } m \leq n \\ 0 & \text { if } m>n\end{cases}
$$

for any pair of integers $m, n$ such that $1 \leq m, n \leq N$. Thus,

$$
\sum_{n \leq M} f(m, n) n^{i t}=\int_{-\infty}^{\infty}\left\{\sum_{n \leq N} f(m, n) n^{i(t+u)}\right\} h(u) M^{-i u} d u
$$

assuming (as we may) that $M$ is an integer. It follows that the left side of (2.18) does not exceed

$$
\begin{aligned}
& \sum_{m} \int_{-\infty}^{\infty} g(t) \int_{-\infty}^{\infty}|h(u)|\left|\sum_{n \leq N} f(m, n) n^{i(t+u)}\right| d u d t \\
& \quad=\sum_{m} \int_{-\infty}^{\infty}\left|\sum_{n \leq N} f(m, n) n^{i \tau}\right|\left\{\int_{-\infty}^{\infty} g(\tau-u)|h(u)| d u\right\} d \tau \\
& \ll T(\log N) \sum_{m} \int_{-\infty}^{\infty}\left|\sum_{n \leq N} f(m, n) n^{i \tau}\right| \min \left\{T^{-1},|\tau|^{-1}, N|\tau|^{-2}\right\} d \tau,
\end{aligned}
$$

where the last step uses (2.19) and the definition of $g$. The desired conclusion now follows by a standard dyadic argument.

3. Proof of Theorem 1.1. In this section we deduce Theorem 1.1 from Theorem 2.1 and Heath-Brown's identity for $\Lambda(n)$. We apply Heath-Brown's identity in the following form (see [4, Lemma 1] or [5, Proposition 13.3] with $k=10)$ : if $n \leq x$, then

$$
\Lambda(n)=\sum_{j=1}^{10}\left(\begin{array}{c}
10 \\
j
\end{array}\right)(-1)^{j} \sum_{\substack{n=m_{1} \cdots m_{2 j} \\
m_{1}, \ldots, m_{j} \leq x^{1 / 10}}} \mu\left(m_{1}\right) \cdots \mu\left(m_{j}\right) \log m_{2 j} .
$$

First, we deal with the possibility that $\mathcal{H}(m, r, Q)$ may contain the principal character $\chi_{0}$ modulo $m$. We have

$$
\sum_{N<n \leq 2 N} \Lambda(n) \chi_{0}(n) n^{-s}=\sum_{N<n \leq 2 N} \Lambda(n) n^{-s}+O\left(L^{2}\right) .
$$

Hence, if $\chi_{0} \in \mathcal{H}(m, r, Q)$, the left side of (1.2) does not exceed

$$
\sum_{\substack{\chi \in \mathcal{H}(m, r, Q) \\ \chi \neq \chi_{0}}} \int_{-T}^{T}\left|\sum_{N<n \leq 2 N} \Lambda(n) \chi(n) n^{-i t}\right| d t+\int_{-T}^{T}\left|\sum_{N<n \leq 2 N} \Lambda(n) n^{-i t}\right| d t+T L^{2} .
$$


Thus, it suffices to establish (1.2) when $\mathcal{H}(m, r, Q)$ is of one of the two types considered in Theorem 2.1.

By (3.1) with $x=2 N$ and a standard splitting argument,

$$
\sum_{N<n \leq 2 N} \Lambda(n) \chi(n) n^{-s} \ll \sum_{\mathbf{M}}\left|\sum_{N<n \leq 2 N} a(n ; \mathbf{M}) \chi(n) n^{-s}\right|,
$$

where $\mathbf{M}$ runs over $O\left(L^{19}\right)$ vectors $\mathbf{M}=\left(M_{1}, \ldots, M_{2 j}\right), j \leq 10$, subject to

$$
M_{1}, \ldots, M_{j} \ll N^{1 / 10}, \quad N \ll M_{1} \cdots M_{2 j} \ll N,
$$

and

$$
a(n ; \mathbf{M})=\sum_{\substack{n=m_{1} \cdots m_{2 j} \\ M_{i}<m_{i} \leq 2 M_{i}}} \mu\left(m_{1}\right) \cdots \mu\left(m_{j}\right)\left(\log m_{2 j}\right) .
$$

Hence, the left side of (1.2) is bounded above by

$$
L^{19} \sum_{\chi \in \mathcal{H}(m, r, Q)} \int_{-T}^{T}\left|\sum_{N<n \leq 2 N} a(n ; \mathbf{M}) \chi(n) n^{-i t}\right| d t
$$

for some fixed choice of $\mathbf{M}$ as above. Thus, if we show that

$$
\sum_{\chi \in \mathcal{H}(m, r, Q)} \int_{-T}^{T}\left|\sum_{n} a(n ; \mathbf{M}) \chi(n) n^{-i t}\right| d t \ll\left(N+H N^{11 / 20}\right) L^{1020},
$$

the desired result (with $C=1100$ ) will follow by Lemma 2.4 .

The Dirichlet polynomial on the left side of (3.2) is the product of $2 j$, $j \leq 10$, Dirichlet polynomials of the form (1.1) with coefficients $a_{n}=\mu(n)$, $a_{n}=1$, or $a_{n}=\log n$. Furthermore, the single logarithmic weight can be removed by partial summation. Therefore, we may assume that

$$
a(n ; \mathbf{M})=L \sum_{\substack{n=m_{1} \cdots m_{2 j} \\ M_{i}<m_{i} \leq M_{i}^{\prime}}} \mu\left(m_{1}\right) \cdots \mu\left(m_{j}\right),
$$

where $M_{i}<M_{i}^{\prime} \leq 2 M_{i}$ (in reality, $M_{i}^{\prime}=2 M_{i}$ except for $i=2 j$ ). We may now assume that $M_{j+1} \leq \cdots \leq M_{2 j}$. We proceed to show that

$$
a(n ; \mathbf{M})=L \sum_{n=n_{1} n_{2} n_{3}} b_{1}\left(n_{1}\right) b_{2}\left(n_{2}\right) b_{3}\left(n_{3}\right),
$$

where the coefficients on the right yield a Dirichlet polynomial (2.1) that satisfies at least one of the hypotheses (i) or (ii) of Theorem 2.1. The analysis involves several cases depending on the sizes of $M_{1}, \ldots, M_{2 j}$.

CASE 1: $M_{2 j} \gg N^{9 / 20}$. Assuming that $j \geq 2$ (the case $j=1$ is similar and easier), we group the variables $m_{1}, \ldots, m_{2 j}$ into $n_{1}, n_{2}, n_{3}$ as follows:

$$
n_{1}=m_{3} \cdots m_{2 j-1}, \quad n_{2}=m_{1} m_{2}, \quad n_{3}=m_{2 j} .
$$


Since $M_{1} \cdots M_{2 j-1} \ll N^{11 / 20}$, this yields a polynomial $F(s, \chi)$ satisfying hypothesis (i) of Theorem 2.1 .

CASE $2: M_{2 j} \ll N^{9 / 20} \ll M_{1} \cdots M_{j} M_{2 j}$. Let $i$ be the least integer for which $M_{1} \cdots M_{i} M_{2 j} \gg N^{9 / 20}$. Since $M_{i} \ll N^{1 / 10}$, we have

$$
N^{9 / 20} \ll M_{1} \cdots M_{i} M_{2 j} \ll N^{11 / 20} .
$$

Hence, the choice

$$
n_{1}=m_{1} \cdots m_{i} m_{2 j}, \quad n_{2}=m_{i+1} \cdots m_{2 j-1}, \quad n_{3}=1
$$

yields an $F(s, \chi)$ that satisfies hypothesis (ii) of Theorem 2.1.

CASE 3: $M_{1} \cdots M_{j} M_{2 j} \ll N^{9 / 20}$. Let $\ell$ be the least positive integer such that

$$
M_{1} \cdots M_{j} M_{\ell} \cdots M_{2 j} \ll N^{9 / 20} .
$$

We consider three subcases.

CAse 3.1: $M_{\ell-1} \cdots M_{2 j} \ll N^{11 / 20}$. Then we can argue similarly to Case 2 to find an $i, 0 \leq i \leq j$, for which

$$
N^{9 / 20} \ll M_{1} \cdots M_{i} M_{\ell-1} \cdots M_{2 j} \ll N^{11 / 20} .
$$

Again, we will have $F(s, \chi)$ that satisfies hypothesis (ii) of Theorem 2.1.

CASE 3.2: $M_{\ell-1} \cdots M_{2 j} \gg N^{11 / 20}$ and $M_{\ell-1} \ll N^{8 / 35}$. Then we define

$$
n_{1}=m_{1} \cdots m_{j} m_{\ell} \cdots m_{2 j}, \quad n_{2}=m_{j+1} \cdots m_{\ell-2}, \quad n_{3}=m_{\ell-1} .
$$

Since $M_{j+1} \cdots M_{\ell-2} \ll N^{9 / 20}$, we again get an $F(s, \chi)$ that satisfies hypothesis (ii) of Theorem 2.1.

CASE 3.3: $M_{\ell-1} \cdots M_{2 j} \gg N^{11 / 20}$ and $M_{\ell-1} \gg N^{8 / 35}$. This may occur only with $\ell=2 j$. Then

$$
M_{1} \cdots M_{2 j-2} \ll N M_{2 j-1}^{-2} \ll N^{19 / 35} \ll N^{11 / 20}, \quad M_{2 j-1} \ll M_{2 j} \ll N^{9 / 20} .
$$

We write

$$
b_{1}(n)=\sum_{n=m_{1} \cdots m_{2 j-2}} \mu\left(m_{1}\right) \cdots \mu\left(m_{j}\right), \quad n_{2}=m_{2 j-1}, \quad n_{3}=m_{2 j},
$$

and we obtain an $F(s, \chi)$ that satisfies hypothesis (i) of Theorem 2.1.

The desired bound (3.2) follows on noting that the arising coefficients satisfy $(2.2)$ with $\kappa, \nu$ for which $c(\kappa, \nu) \leq c(18,2)=1012$.

4. Exponential sums twisted by characters. In this section we estimate the exponential sum

$$
W(\beta, \chi)=\sum_{N<p \leq 2 N}(\log p) \chi(p) e\left(\beta p^{k}\right)
$$


where $k$ is a positive integer, $\beta$ is "small", and $\chi$ is Dirichlet character. Such exponential sums arise in dealings with the major arcs in the WaringGoldbach problem and related questions. In particular, in the proof of Theorem 1.3 we need the case $k=1$ of our estimates.

Lemma 4.1. Suppose that $N \geq 2$ and $0 \leq \Delta \leq N^{1-k}$. Suppose also that $\mathcal{H}(m, r, Q)$ is a set of characters as in Theorem 1.1 and $W(\beta, \chi)$ is defined by (4.1). Then

$$
\sum_{\chi \in \mathcal{H}(m, r, Q)} \max _{\Delta \leq|\beta| \leq 2 \Delta}|W(\beta, \chi)| \ll T_{0}^{-1 / 2} L^{C+1}\left(N+H N^{11 / 20}\right),
$$

where $T_{0}=1+\Delta N^{k}, H=m r^{-1} Q^{2} T_{0}, L=\log N$, and $C$ is the constant appearing in Theorem 1.1.

Proof. We first replace $W(\beta, \chi)$ by the exponential sum

$$
\widetilde{W}(\beta, \chi)=\sum_{N<n \leq 2 N} \Lambda(n) \chi(n) e\left(\beta n^{k}\right)
$$

using the fact that

$$
W(\beta, \chi)=\widetilde{W}(\beta, \chi)+O\left(N^{1 / 2}\right) .
$$

By Perron's formula [5, Proposition 5.54], for $N<M \leq 2 N$,

$$
\begin{aligned}
\sum_{N<n \leq M} \Lambda(n) \chi(n) & \\
& =\frac{1}{2 \pi i} \int_{b-i T_{1}}^{b+i T_{1}} F(s, \chi) \frac{M^{s}-N^{s}}{s} d s+O\left(\frac{N L^{2}}{1+T_{1}\|M\|}\right),
\end{aligned}
$$

where $0<b<(\log N)^{-1}, T_{1}=(H N)^{10},\|M\|$ is the distance from $M$ to the nearest integer, and

$$
F(s, \chi)=\sum_{N<n \leq 2 N} \Lambda(n) \chi(n) n^{-s}
$$

Hence, by partial summation,

$$
\widetilde{W}(\beta, \chi)=\frac{1}{2 \pi i} \int_{b-i T_{1}}^{b+i T_{1}} F(s, \chi) V(s, \beta) d s+O(1),
$$

where

$$
V(s, \beta)=\int_{N}^{2 N} y^{s-1} e\left(\beta y^{k}\right) d y .
$$

By [5, Lemma 8.10], for $\Delta \leq|\beta| \leq 2 \Delta$,

$$
V(\sigma+i t, \beta) \ll N^{\sigma} \min \left\{T_{0}^{-1 / 2}, \sup _{N \leq y \leq 2 N}\left|t+2 k \pi \beta y^{k}\right|^{-1}\right\} .
$$


Combining (4.5) and (4.6) and letting $b \downarrow 0$, we obtain

$$
\widetilde{W}(\beta, \chi) \ll T_{0}^{1 / 2} \int_{-T_{1}}^{T_{1}}|F(i t, \chi)| \frac{d t}{T_{0}+|t|}+1 .
$$

Recalling (4.3), we deduce that the left side of (4.2) is bounded above by

$$
L T_{0}^{1 / 2} T^{-1} \sum_{\chi \in \mathcal{H}(m, r, Q)} \int_{-T}^{T}|F(i t, \chi)| d t+|\mathcal{H}| N^{1 / 2},
$$

for some $T$ in the range $T_{0} \leq T \leq T_{1}$. The result now follows from (1.2).

We now define the exponential integral

$$
v(\beta ; X)=\int_{X}^{2 X} e\left(\beta y^{k}\right) d y .
$$

Lemma 4.2. Suppose that $N \geq 2,1 \leq Q \leq N$, and $0 \leq \Delta \leq N^{1-k}$. Let $W(\beta, \chi)$ be defined by (4.1). Then, for any fixed $A>0$ and $\delta>0$,

$$
\sum_{Q<q \leq 2 Q} \sum_{\chi \bmod q}^{*} \max _{\Delta \leq|\beta| \leq 2 \Delta}|W(\beta, \chi)| \ll N Q^{\delta} L^{-A}+Q^{2} T_{0}^{1 / 2} N^{11 / 20} L^{C+1},
$$

where $T_{0}=1+\Delta N^{k}, L=\log N$, and $C$ is the constant appearing in Theorem 1.1. Furthermore, for any fixed $A>0$ we have

$$
W\left(\beta, \chi^{0}\right)-v(\beta ; N) \ll N L^{-A}+T_{0}^{1 / 2} N^{11 / 20} L^{C+1},
$$

where $v(\beta ; N)$ is defined by (4.8) and $\chi^{0}$ is the trivial character. In both (4.9) and (4.10) the implied constants may depend on $A$, and the implied constant in (4.9) may also depend on $\delta$.

Proof. The first claim follows from Lemma 4.1 and the Siegel-Walfisz theorem in the form of $[5,(5.79)]$. Put $B=\left(2+\delta^{-1}\right)(A+C+1)$. If $Q \geq L^{B}$ or $\Delta \geq L^{B} N^{-k}$, we have

$$
N T_{0}^{-1 / 2} L^{C+1} \ll N Q^{\delta} L^{-A}
$$

and (4.9) follows from (4.2) with $m=r=1$. On the other hand, if $Q \leq L^{B}$ and $\Delta \leq L^{B} N^{-k}$, we find by partial summation that the left side of (4.9) is bounded above by

$$
L^{3 B+1} \max _{N<N_{1} \leq 2 N}\left|\sum_{N<p \leq N_{1}} \chi(p)\right| \ll N L^{-A},
$$

by the aforementioned version of the Siegel-Walfisz theorem.

The proof of the second claim is similar, except that it appeals to the case $m=r=Q=1$ of Lemma 4.1 and to the prime number theorem (which is why we need to include the term $v(\beta ; N)$ on the left side of $(4.10))$. 
Lemma 4.3. Suppose that $N \geq 2$ and $N^{-k} \leq \Delta \leq N^{1-k}$. Suppose also that $\mathcal{H}(m, r, Q)$ is a set of characters as in Theorem 1.1 and $W(\beta, \chi)$ is defined by (4.1). Then

$$
\sum_{\chi \in \mathcal{H}(m, r, Q)}\left\{\int_{-\Delta}^{\Delta}|W(\beta, \chi)|^{2} d \beta\right\}^{1 / 2} \ll N^{-k / 2} L^{C+1}\left(N+H N^{11 / 20}\right),
$$

where $H=m r^{-1} Q^{2} \Delta N^{k}, L=\log N$, and $C$ is the constant from Theorem 1.1 .

Proof. By [10, Lemma 1.9], we have

$$
\begin{aligned}
\int_{-\Delta}^{\Delta}|W(\beta, \chi)|^{2} d \beta & \ll \Delta^{2} \int_{-\infty}^{\infty}\left|\sum_{u(y)<p \leq v(y)}(\log p) \chi(p)\right|^{2} d y \\
& \ll \Delta^{2} N^{k}\left|\sum_{M<n \leq M+Y} \Lambda(n) \chi(n)\right|^{2}+\Delta^{2} N^{k+1},
\end{aligned}
$$

where $u(y)=\max \left(N, y^{1 / k}\right), v(y)=\min \left(2 N,\left(y+(2 \Delta)^{-1}\right)^{1 / k}\right)$, and

$$
N<M \leq 2 N, \quad Y \ll \Delta^{-1} N^{1-k} .
$$

Without loss of generality, we may assume that the distance from $M$ to the nearest integer is $1 / 2$ and that $Y$ is an integer. We then appeal to Perron's formula to derive

$$
\sum_{M<n \leq M+Y} \Lambda(n) \chi(n) \ll\left|\int_{b-i T_{1}}^{b+i T_{1}} F(s, \chi) \frac{(M+Y)^{s}-M^{s}}{s} d s\right|+1,
$$

where $0<b<L^{-1}, T_{1}=(H N)^{10}$, and $F(s, \chi)$ is the Dirichlet polynomial appearing in the proof of Lemma 4.1. Hence, as in that proof,

$$
\sum_{M<n \leq M+Y} \Lambda(n) \chi(n) \ll \int_{-T_{1}}^{T_{1}}|F(i t, \chi)| \frac{d t}{T_{0}+|t|}+1,
$$

where $T_{0}=\Delta N^{k}$. By (4.12) and (4.14), the left side of (4.11) is bounded above by

$$
\Delta N^{k / 2} L T^{-1} \sum_{\chi \in \mathcal{H}(m, r, Q)} \int_{-T}^{T}|F(i t, \chi)| d t+H N^{(1-k) / 2},
$$

where $T$ is subject to $T_{0} \leq T \leq T_{1}$. The result now follows from (1.2).

5. Proof of Theorem 1.3. Since the proof follows closely the proof of the main result in [7], we only describe the necessary changes. Let $N$ be a large parameter chosen as in [7, Lemma 2.3] and set

$$
P=(N / B)^{9 / 20}, \quad L=\log N, \quad Q=N /\left(P L^{2}\right) .
$$


We note that the improvement on the result of Liu and Tsang arises from the choice of $P$ in (5.1): the respective choice in [7] is $P=(N / B)^{2 / 5}$ (see $[7,(2.1)])$. In order to justify the analysis in [7] for this larger value of $P$, we need appropriate variants of [7, Lemmas 3.2 and 3.3]. If we weaken the bound in part (i) of [7, Lemma 3.3] to

$$
J_{j}(R) \ll \tau(\varpi) R^{-1 / 9} N\left|a_{j}\right|^{-1} L^{c},
$$

then the modified lemma (which suffices for the proof of Theorem 1.3) holds with $P=(N / B)^{9 / 20}$.

Now, let $N_{j}=N /\left|a_{j}\right|, N^{1 / 10} \leq R \leq P$, and $g, D$ be positive integers. Define

$$
K_{j}(g ; R)=\sum_{R<r \leq 2 R} \frac{\sqrt{([g, r], D)}}{[g, r]} \sum_{\chi \bmod r}^{*}\left(\int_{-1 /(R Q)}^{1 /(R Q)}\left|W_{j}\left(a_{j} \beta, \chi\right)\right|^{2} d \beta\right)^{1 / 2},
$$

where $W_{j}(\beta, \chi)$ is the sum (4.1) with $N=N_{j}$ and $k=1$. In order to prove [7, Lemma 3.2] with $P$ as in (5.1), we need to show that

$$
K_{j}(g ; R) \ll g^{-1} \sqrt{(g, D)} \tau(g D)^{2} N_{j} N^{-1 / 2} L^{c}
$$

for some absolute constant $c$. By $[7,(5.20)]$,

$$
K_{j}(g ; R) \ll \frac{\sqrt{(g, D)}}{g R} \sum_{\substack{d \mid g D \\ d \leq 2 R}} d \tau(d) \widetilde{K}_{j}(d ; R),
$$

where

$$
\widetilde{K}_{j}(d ; R)=\sum_{\chi \in \mathcal{H}(1, d, 2 R)}\left(\int_{-1 /(R Q)}^{1 /(R Q)}\left|W_{j}\left(a_{j} \beta, \chi\right)\right|^{2} d \beta\right)^{1 / 2} .
$$

By Lemma 4.3 with $k=1$,

$$
\begin{aligned}
\widetilde{K}_{j}(d ; R) & \ll\left|a_{j}\right|^{-1 / 2} \sum_{\chi \in \mathcal{H}(1, d, 2 R)}\left(\int_{-\left|a_{j}\right| /(R Q)}^{\left|a_{j}\right| /(R Q)}\left|W_{j}(\beta, \chi)\right|^{2} d \beta\right)^{1 / 2} \\
& \ll N^{-1 / 2} L^{C+1}\left(N_{j}+H_{j} N_{j}^{11 / 20}\right),
\end{aligned}
$$

where $C$ is the constant appearing in Theorem 1.1 and

$$
H_{j}=d^{-1} R^{2}\left(\left|a_{j}\right| /(R Q)\right) N_{j} \ll d^{-1} P R L^{2} \ll d^{-1} R N_{j}^{9 / 20} L^{2} .
$$

Thus,

$$
\widetilde{K}_{j}(d ; R) \ll N_{j} N^{-1 / 2} L^{C+3}(R / d+1) .
$$

Clearly, this inequality and (5.3) imply (5.2). This completes the proof of the theorem. 
Acknowledgements. The authors would like to thank the referee for his/her careful reading of the manuscript and several suggestions that helped improve the exposition.

\section{References}

[1] A. Baker, On some diophantine inequalities involving primes, J. Reine Angew. Math. 228 (1967), 166-181.

[2] K. K. Choi, A numerical bound for Baker's constant: some explicit estimates for small prime solutions of linear equations, Bull. Hong Kong Math. Soc. 1 (1997), $1-19$.

[3] K. K. Choi, M. C. Liu and K. M. Tsang, Conditional bounds for small prime solutions of linear equations, Manuscripta Math. 74 (1992), 321-340.

[4] D. R. Heath-Brown, Prime numbers in short intervals and a generalized Vaughan identity, Canad. J. Math. 34 (1982), 1365-1377.

[5] H. Iwaniec and E. Kowalski, Analytic Number Theory, Amer. Math. Soc., 2004.

[6] H. Z. Li, Small prime solutions of some ternary linear equations, Acta Arith. 98 (2001), 293-309.

[7] J. Y. Liu and K. M. Tsang, Small prime solutions of ternary linear equations, ibid. 118 (2005), $79-100$.

[8] M. C. Liu and K. M. Tsang, Small prime solutions of linear equations, in: Théorie des nombres, de Gruyter, 1989, 595-624.

[9] M. C. Liu and T. Z. Wang, A numerical bound for small prime solutions of some ternary linear equations, Acta Arith. 86 (1998), 343-383.

[10] H. L. Montgomery, Topics in Multiplicative Number Theory, Springer, 1971.

Department of Mathematics and Statistics

Simon Fraser University

Burnaby, Canada V5A 1S6

E-mail:kkchoi@math.sfu.ca
Department of Mathematics Towson University 8000 York Road

Towson, MD 21252, U.S.A. E-mail: akumchev@towson.edu

Received on 3.1.2005

and in revised form on 8.3.2006 